\title{
The acoustic 2-D wave equation in multi-scale transform domains
}

\author{
M. V. C. Henriques ${ }^{\diamond}$, G. Corso*†, J. E. Freitas*, F. A. Moura*, L. S. Lucena* \\ $\diamond$ Departamento de Ciências Exatas e Tecnologia da Informação, Universidade Federal Rural do Semi-Árido - UFERSA \\ 59515-000 Angicos, RN, Brazil. \\ *Programa de Pós-Graduação em Ciência e Engenharia do Petróleo, Universidade Federal do Rio Grande do Norte - UFRN, \\ 59078-970, Natal, RN, Brazil. \\ $\dagger$ Departamento de Biofísica e Farmacologia, Centro de Biociências, Universidade Federal do Rio Grande do Norte - UFRN \\ 59078-970, Natal, RN, Brazil.
}

Copyright 2019, SBGf - Sociedade Brasileira de Geofísica.

This paper was prepared for presentation at the $16^{\text {th }}$ International Congress of the Brazilian Geophysical Society, held in Rio de Janeiro, Brazil, 19-22 August, 2019.

Contents of this paper were reviewed by the Technical Committee of the $16^{\text {th }}$ International Congress of the Brazilian Geophysical Society and do not necessarily represent any position of the SBGf, its officers or members. Electronic reproduction or storage of any part of this paper for commercial purposes without the written consent of the Brazilian Geophysical Society is prohibited.

\section{Abstract}

The computational cost of full wave inversion FWI is high. In this context, sparse representations are an alternative strategy to face this problem. In the present work we deal with the wave dynamics in a homogeneous space for a sparse representation. We present a mathematical framework that transform the space time FWI wave forms of the wave equation into a sparse representation. In addition, we work a fast and efficient dynamical evolution for the coefficients of the wave forms in this sparse representation. We successfully tested the evolution of the coefficients in a homogeneous model.

\section{Introduction}

The gas and oil industry is one of leading economical activities in the world, King (1997). A major challenge in gas and oil geophysics exploration is to obtain good images of the subsurface, Virieux and Operto (2009). In fact, a great effort of science and engineering is to develop adequate tools of subsurface imaging. In recent years, the full wave inversion FWI method is successfully being applied to the seismic image field, Fichtner (2010).

The experimental background of the FWI is the standard reflection seismic exploration: an explosive source generates a pulse wave that propagates in the subsurface, reflect in the geologic layers and is captured in the geophones of hydrophones Yilmaz (2001). The challenge of the FWI technique consists in, with help of the physics laws of wave propagation, to compute the wave forms that travel in the subsurface. The computational cost of the FWI is huge, the used memory during the FWI processing makes this enterprise one of the highest memory consumption activities of computational industry, Herrmann et al. (2012).

The concept of sparse representations is used in mathematics to describe peculiar data transformations, Stéphane (2009). When is is possible to transform a massive data set into another data with significant lesser memory, but keeping the relevant information, we say that the new compressed data is in a sparse representation. In other words, the transformation map the initial large data into a sparse representation. The most common example of sparse representation is the Fourier transform. An additional example is the wavelet transformation that is used to build the jpeg compressed file method. Our physical mathematical manuscript is inscribed in the context of wave propagation in sparse representations.

One more word about the FWI method. The FWI was initially posed in the time domain representation, Tarantola (1984) because the wave equation is written for a traveling wave in time and space. A further advance on the FWI method transform the FWI in the frequency domain, Sirgue and Pratt (2004), using the usual Fourier transform of the wave equation. However, a more general representation of the $\mathrm{FWI}$ method, like the wavelet or curvelet representations, suffers from an explicit matrix wave propagator. The objective of this work is to explore wave propagation in space representation for any well behaved mathematical transformation.

To illustrate our methodology we exemplify our results using curvelet transformation, Devaney (2012). We hope that a representation based on tight frames like Curvelets would be suitable to simulate the wave propagation in an intuitive way. The curvelets are mathematical objects that, besides its multi-scale characteristics inherited from the wavelets, have strong dependence on orientation, represent. So, they can sparsely represent the anisotropy of wave patterns. In the reference Candes and Demanet (2005) it is demonstrated that the solution of a large class of wave equations are optimally sparse in the curvelet domain in the case of smooth variations of the coefficients of the wave equations. However, we believe that the sparsity remains even when the physical properties of the medium of propagation vary sharply. This is the case of the geological medium studied in geophysics.

The seismic explorations is an area that deals with a huge amount of data. The search for sparse representations is a challenge in this field. In this manuscript we introduce a matrix that propagates the wave for a general mathematical transformation. We believe that our approach should open a new perspective in the FWI technique. The rest of the paper is organized as: in section 2 we present the mathematical framework that deals with wave 
equation, sparse representations and matrix propagation. In section 3 we exemplify our methodology using curvelet transformation. Finally in section 4 we conclude the work and put the results in a broad perspective.

\section{Mathematical Framework}

\section{Tight Frames}

Frame theory (Duffin and Schaeffer, 1952) seeks to establish conditions under which a function $f$ in a Hilbert space $\mathscr{H}$ can be perfectly recovered from a family of vectors $\left\{\phi_{\alpha}\right\}_{\alpha \in \Omega}$, with $\Omega$ representing a set of discrete indexes.

Let $f$ be a function with finite energy in a Hilbert space $\mathscr{H}$, and let $\left\{\phi_{\alpha}\right\}$ be a frame that can span the space $\mathscr{H}$. One call $\left\{\phi_{\alpha}\right\}$ a tight frame if the inner product of $f$ with all the elements of the frame keeps the energy finite:

$$
A\|f(x)\|=\sum_{\alpha \in \Omega}\left|\left\langle f \mid \phi_{\alpha}\right\rangle\right|^{2}
$$

where $A>0$ is a constant, $\|\cdot\|$ is a suitable norm for the Hilbert space, $\langle\cdot \mid \cdot\rangle$ denotes the inner product and the discrete indexes $\alpha$ can assume any of the values allowed in $\Omega$. One could say that $\left\{\phi_{\alpha}\right\}$ can represent, even if in an unorthogonal way, i.e., redundantly, any function of the space $\mathscr{H}$ :

$$
f=\sum_{\alpha \in \Omega}\left\langle f \mid \phi_{\alpha}\right\rangle \phi_{\alpha}
$$

An advantage of such representation is immediate: let a family of functions $\{f\}$ represent a physical signal, for example, acoustic wave fields. If one finds a frame on which this family $\{f\}$ can be sparsely represented, a signal could be easily compressed with little loss of information as follows:

$$
\widetilde{f}=\sum_{\alpha \in \text { Relevant }}\left\langle f \mid \phi_{\alpha}\right\rangle \phi_{\alpha}
$$

by discarding the negligible coefficients according to an established threshold.

\section{Transforming the wave equation}

The homogeneous (without sources) acoustic wave equation in a medium with constant propagation velocity $v$ is given by:

$$
v^{2} \nabla^{2} u(\boldsymbol{x}, t)-\partial_{t}^{2} u(\boldsymbol{x}, t)=0
$$

where $\nabla^{2}$ is the Laplacian, $\partial_{t}^{2}$ is the second time derivative operator and $u$ is a scalar function that represents the wave field, Feynman et al. (1965).

Let the operator $\mathscr{F}$ be the frame transform that projects this equation on the tight frame $\{\phi\}$. Applying $\mathscr{F}$ to the wave equation (4):

$$
\mathscr{F}\left\{v^{2} \nabla^{2} u(\boldsymbol{x}, t)-\partial_{t}^{2} u(\boldsymbol{x}, t)\right\}=0
$$

If the operator $\mathscr{F}$ is linear, we have:

$$
v^{2} \mathscr{F}\left\{\nabla^{2} u(\boldsymbol{x}, t)\right\}-\mathscr{F}\left\{\partial_{t}^{2} u(\boldsymbol{x}, t)\right\}=0
$$

In addition, the $\partial_{t}^{2}$ operator on the tight frame fulfill the condition:

$$
\begin{aligned}
\mathscr{F}\left\{\partial_{t}^{2} u(\boldsymbol{x}, t)\right\}(\alpha) & =\left\langle\partial_{t}^{2} u(\boldsymbol{x}, t) \mid \phi_{\alpha}(\boldsymbol{x})\right\rangle \\
& =\left\langle\partial_{t}^{2} u \mid \phi_{\alpha}\right\rangle \\
& =\partial_{t}^{2}\left\langle u \mid \phi_{\alpha}\right\rangle \\
& =\partial_{t}^{2} c_{u}(\alpha)
\end{aligned}
$$

where we label $\left\langle u \mid \phi_{\alpha}\right\rangle=c_{u}(\alpha)$, the coefficient of the field $u$ for the frame element $\phi_{\alpha}$

\section{The Laplacian operator in a tight frame.}

Let $\left\{\phi_{\alpha}\right\}_{\alpha \in \Omega}$ be a tight frame of the Hilbert space $\mathscr{H}$ of the wavefunctions in 2 dimensions:

$$
\begin{aligned}
\mathscr{F}\left\{\nabla^{2} u(\boldsymbol{x}, t)\right\}(\alpha) & =\left\langle\nabla^{2} u(\boldsymbol{x}, t) \mid \phi_{\alpha}(\boldsymbol{x})\right\rangle \\
& =\left\langle\nabla^{2} u \mid \phi_{\alpha}\right\rangle \\
& =\int_{-\infty}^{\infty} \int_{-\infty}^{\infty} \nabla^{2} u(x, y, t) \phi_{\alpha}(x, y) \mathrm{d} x \mathrm{~d} y
\end{aligned}
$$

Taking into account that $\phi_{\alpha}$ and $u$ are continuous functions, one can apply the two-dimensional version of the second Green identity:

$$
\int_{s}\left(\phi_{\alpha} \nabla^{2} u-u \nabla^{2} \phi_{\alpha}\right) \mathrm{d} s=\oint_{c}\left(\phi_{\alpha} \nabla u-u \nabla \phi_{\alpha}\right) \cdot \boldsymbol{n} \mathrm{d} l
$$

in which $c$ represents the path along the boundary of an arbitrary surface $s$, and $\boldsymbol{n}$ is the unit normal vector of de line element $\mathrm{d} l$ (Courant and Hilbert, 1989). If one has a tight frame composed by localized elements $\phi_{\alpha}$ that have finite values on a small vicinity and decay rapidly for long distances, one can take the integration surface large enough such that, at the boundary of the surface $s, \phi_{\alpha}(s)=0$ and $\nabla \phi_{\alpha}(s)=0$ and, as a consequence, the second integral equals zero:

$$
\int_{s}\left(\phi_{\alpha} \nabla^{2} u-u \nabla^{2} \phi_{\alpha}\right) \mathrm{d} s=0
$$

One can imagine that the surface covered by the integral in (10) is so big that the value of the integral is practically the same as that of the infinite integral in (8), which approximately justifies:

$$
\int_{-\infty}^{\infty} \int_{-\infty}^{\infty}\left(\phi_{\alpha} \nabla^{2} u-u \nabla^{2} \phi_{\alpha}\right) \mathrm{d} x \mathrm{~d} y=0
$$

Consequently:

$$
\begin{aligned}
\int_{-\infty}^{\infty} \int_{-\infty}^{\infty} \phi_{\alpha} \nabla^{2} u \mathrm{~d} x \mathrm{~d} y & =\int_{-\infty}^{\infty} \int_{-\infty}^{\infty} u \nabla^{2} \phi_{\alpha} \mathrm{d} x \mathrm{~d} y \\
\left\langle\nabla^{2} u \mid \phi_{\alpha}\right\rangle & =\left\langle u \mid \nabla^{2} \phi_{\alpha}\right\rangle \\
\mathscr{F}\left\{\nabla^{2} u\right\}(\alpha) & =\left\langle u \mid \nabla^{2} \phi_{\alpha}\right\rangle
\end{aligned}
$$

Let the frame $\left\{\phi_{\alpha}\right\}_{\alpha \in \Omega}$ be a complete representation, even if it is not orthonormal. One can write:

$$
\begin{aligned}
f & =\sum_{\alpha \in \Omega}\left\langle f \mid \phi_{\alpha}\right\rangle \phi_{\alpha} \\
& =\sum_{\alpha \in \Omega} c_{f}(\alpha) \phi_{\alpha}
\end{aligned}
$$


for all $f \in \mathscr{H}$, and where $\Omega$ is the set of all $\alpha$ of indexes of the frame $\left\{\phi_{\alpha}\right\}$. As a consequence, the completeness relation is valid:

$$
\sum_{\alpha \in \Omega}\left|\phi_{\alpha}\right\rangle\left\langle\phi_{\alpha}\right|=1
$$

One can insert the relation (16) in (14):

$$
\begin{aligned}
\mathscr{F}\left\{\nabla^{2} u\right\}(\alpha) & =\left\langle u\left|\left(\sum_{\alpha^{\prime} \in \Omega}\left|\phi_{a^{\prime}}\right\rangle\left\langle\phi_{a^{\prime}}\right|\right)\right| \nabla^{2} \phi_{\alpha}\right\rangle \\
& =\sum_{\alpha^{\prime} \in \Omega}\left\langle u \mid \phi_{a^{\prime}}\right\rangle\left\langle\phi_{a^{\prime}} \mid \nabla^{2} \phi_{\alpha}\right\rangle \\
& =\sum_{\alpha^{\prime} \in \Omega} c_{u}\left(\alpha^{\prime}\right)\left\langle\phi_{a^{\prime}} \mid \nabla^{2} \phi_{\alpha}\right\rangle
\end{aligned}
$$

If we choose our frame to be real-valued $(\phi(x, y) \in \mathbb{R})$, the equality $\left\langle\phi_{\alpha} \mid \nabla^{2} \phi_{\alpha}\right\rangle=\left\langle\nabla^{2} \phi_{\alpha} \mid \phi_{\alpha}\right\rangle$ holds, and:

$$
\mathscr{F}\left\{\nabla^{2} u\right\}(\alpha)=\sum_{\alpha^{\prime} \in \Omega} c_{u}\left(\alpha^{\prime}\right)\left\langle\nabla^{2} \phi_{\alpha} \mid \phi_{\alpha^{\prime}}\right\rangle
$$

Finally, the homogeneous wave equation expressed by means of coefficients $c_{u}(\alpha)$ can be written as:

$$
v^{2} \sum_{\alpha^{\prime} \in \Omega} c_{u}\left(\alpha^{\prime}\right)\left\langle\nabla^{2} \phi_{\alpha} \mid \phi_{\alpha^{\prime}}\right\rangle-\partial_{t}^{2} c_{u}(\alpha)=0
$$

\section{Curvelets tight frame}

Curvelets are a multiscale system of representation in which the elements are highly anisotropic at fine scales, with effective support shaped according to the parabolic scaling principle, width $\approx$ length $^{2}$, at fine scales. The curvelets have been shown to be optimally sparse in the representation of propagating wavefields, Candes and Demanet (2005).

Curvelets are oscillatory and highly localized in space, decaying rapidly at long distances from their center. These properties make curvelets suitable as a tight frame in our work. Each curvelet $\left\{\phi_{\alpha}\right\}$ at scale $a$ is constructed from a "mother" curvelet $\phi_{a}$ by scaling, shifting and rotating.

\section{Discretization of the equation}

In order to implement the equation (19) in a computer code we have to perform an adequate discretization. The time derivative of the frame coefficients of the wavefield can be approximated by the finite differences approach:

$$
\partial_{t}^{2} c_{u}(\alpha) \approx \frac{c_{u}^{n+1}(\alpha)-2 c_{u}^{n}(\alpha)+c_{u}^{n-1}(\alpha)}{\Delta t^{2}}
$$

So, equation (19) turns into

$$
\begin{aligned}
v^{2} \sum_{\alpha^{\prime} \in \Omega} c_{u}^{n}\left(\alpha^{\prime}\right) & \left\langle\nabla^{2} \phi_{\alpha} \mid \phi_{\alpha^{\prime}}\right\rangle \\
& -\frac{\left(c_{u}^{n+1}(\alpha)-2 c_{u}^{n}(\alpha)+c_{u}^{n-1}(\alpha)\right)}{\Delta t^{2}}=0
\end{aligned}
$$

Rearranging,

$$
\begin{aligned}
& c_{u}^{n+1}(\alpha)= \\
& -c_{u}^{n-1}(\alpha)+2 c_{u}^{n}(\alpha)+v^{2} \Delta t^{2} \sum_{\alpha^{\prime} \in \Omega} c_{u}^{n}\left(\alpha^{\prime}\right)\left\langle\nabla^{2} \phi_{\alpha} \mid \phi_{\alpha^{\prime}}\right\rangle
\end{aligned}
$$

\section{Numerical Experiment}

The numerical experiment that follows illustrate the mathematical framework developed in the previous section. Namely, we tested the adequacy of equation (19) for the time evolution of the coefficients of a sparse representation. To that end, we implemented on the discretized version of this relation, equation (22), that is designed to be implemented in computational algorithms. As stated before, we employed the curvelet transform as a study case because of its ability to represent the wavefield sparsely.

In our numerical simulations we modeled the propagation of an acoustic wave with a pulse source in a 2D homogeneous medium. Moreover, we employ a Ricker wavelet, Ricker (1953), as the source pulse. The curvelets are labeled, in the frequency domain, by $\phi_{j, l, k}$ where $j$ and $l$ are integer indexes that define the scale and orientation, respectively, and $\boldsymbol{k}$ is a integer 2-tuple that defines the twodimensional spatial localization; these three parameters uniquely identify the curvelet as the Fourier transform of the original curvelets in real space.

Results of our tests are shown in figure 1. Figure 1(a) shows a snapshot of the modeled waveform using the standard wave equation (4). In figure 1(b) we depict the same waveform using the dynamical temporal evolution based on the curvelet coefficient evolution, equation (22). To compare both approaches we compute the differences between them and plotted the result in figure 1(c).

To better showcase the advantage of this curvelet-based time evolution algorithm we show in figure 2 some examples of the projection of the Laplacian of a curvelet in the base $\nabla^{2} \phi_{n}$ on another curvelet $\phi_{m}$. We chose elements on the scale $j=3$ and orientations $l$, labeled $\phi_{j, l}$, to project on the curvelet with the same scale and close orientation. These projections $\left\langle\nabla^{2} \phi_{j, l} \mid \phi_{j^{\prime}, l^{\prime}}\right\rangle$ are the central element of equations (19) or (22) that describe the time dynamical evolution of the coefficient of $\phi_{j, l}$ of the wavefield represented in the curvelet tight frame.

Figure 2 shows, as an example, the numerical results of the inner product $\left\langle\nabla^{2} \phi_{3, l} \mid \phi_{3,4}\right\rangle$. In fact, the projection of the Laplacians orientations close to $l=4$ concentrate almost all the energy while the other coefficients carry only a small amount of energy. As one can see, $\left(\nabla^{2} \phi_{3,3}, \nabla^{2} \phi_{3,4}\right.$ and $\left.\nabla^{2} \phi_{3,5}\right)$ are the elements that concentrate most of energy in the projection. We remind that equation (22) represents a sum over all $k_{i}$ and $k_{j}$ elements, but the panels in figure 2 make it evident by the concentration of energy over a few projections $\left\langle\nabla^{2} \phi_{i, j} \mid \phi_{3,4}\right\rangle$ that to calculate the evolution of the coefficient of $\phi_{3,4}$ it would be enough to compute just a few projections on coefficients with neighboring wave vectors and neglect the others.

\section{Final Remarks}

In this manuscript we developed a mathematical formulation for the dynamical evolution of a propagating acoustic wave that is adequate for sparse representations. In fact, it is adequate for any linear transformation that fulfill proper convergence conditions. This framework is specially fitted for the FWI methodology as it is well suited to promote savings in memory and processing time, since the wavefields can be propagated directly in a compacted 
(a)
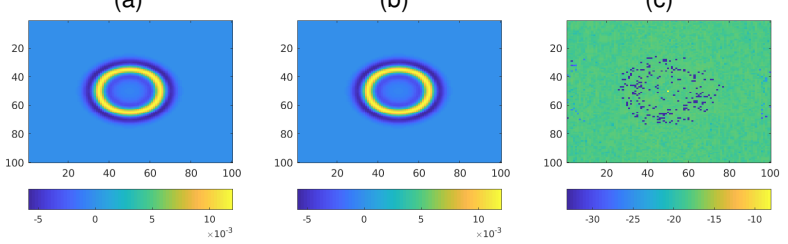

Figure 1: Comparison between two wavefields: the first, shown in (a), is obtained using the standard numerical solution process via finite differences of the wave equation (4). In the panel (b) it is shown the result of the dynamical evolution using the propagation of the curvelet coefficients as in equation (22). In (c) it is plotted the difference between the two figures.

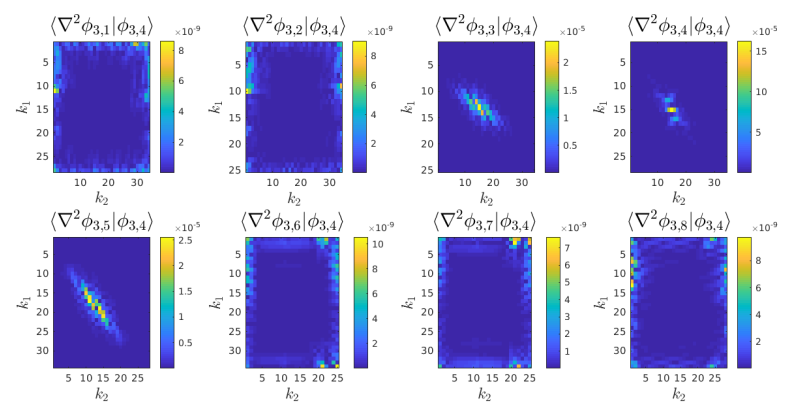

Figure 2: Inner product between the curvelet, $\phi_{3,4}$, and neighbour curvelets Laplacian at the same scale $3, \nabla^{2} \phi_{3, j}$, with adjacent orientations.

form.

The computational implementation of the mathematical principles was demonstrated to be successful in two ways. First, we confirmed that a curvelet-based representation is indeed a sparse representation for wave forms over time, only a few coefficients are necessary to describe typical wave forms; this fact is frequently referenced in the literature, Candes et al. (2006). The second, and more important point, is that the time evolution of the wave forms is well described by our algorithms. The dynamical evolution of the curvelet coefficients is quite similar to the dynamical evolution of the wave equation itself.

We remark that this paper is a proof of concept for a work in progress. The numerical demonstration in this paper was carried out for a homogeneous medium as a test case. At this moment we are working to account for discontinuities and inhomogeneity in the medium in the mathematical model to make the methodology more realistic to the $\mathrm{FWI}$ application, while still preserving the advantageous sparsity of representation.

The number of possible curvelets to be used as mother curvelets in the creation of a base are endless, so we direct our efforts in creating a specifc curvelet tight frame that allows us to neglect most of the terms involving the laplacian projections of the base elements. Such a family of curvelets would allow us to calculate the propagation in an already compacted form, with interesting savings in memory used for storage of the wavefields and processing time in modeling the time evolution.
To summarize the work: FWI imaging is computationally costly, sparse representations can save memory and processing time, we developed mathematical tools that skip the usual wave equation space time evolution by working directly with the coefficients of a sparse representation.

\section{ACKNOWLEDGMENTS}

The authors acknowledge the support of Conselho Nacional de Desenvolvimento Científico e Tecnológico, $\mathrm{CNPq}$ - Brazil. The authors also gratefully acknowledge support from Shell Brasil through the "New Methods for Full Waveform Inversion" project at Federal University of Rio Grande do Norte (UFRN) and the strategic importance of the support given by ANP through the R\&D levy regulation.

\section{References}

P. R. King, Physics boosts oil production, Physics World 10 (1997) 33-37.

J. Virieux, S. Operto, An overview of full-waveform inversion in exploration geophysics, Geophysics 74 (2009) WCC1-WCC26.

A. Fichtner, Full Seismic Waveform Modelling and Inversion, Springer Verlag, 2010.

O. Yilmaz, Seismic data analysis: Processing, inversion, and interpretation of seismic data., Society of exploration geophysicists, 2001.

F. J. Herrmann, M. P. Friedlander, O. Yilmaz, Fighting the curse of dimensionality: Compressive sensing in exploration seismology, IEEE Signal Processing Magazine 29 (2012) 88-100.

M. Stéphane, Chapter 1 - sparse representations, in: M. Stéphane (Ed.), A Wavelet Tour of Signal Processing (Third Edition), Academic Press, Boston, third edition edition, 2009, pp. 1 - 31.

A. Tarantola, Inversion of seismic reflection data in the acoustic approximation, Geophysics 49 (1984) 1259 1266.

L. Sirgue, R. G. Pratt, Efficient waveform inversion and imaging: A strategy for selecting temporal frequencies, Geophysics 69 (2004) 231-248.

A. Devaney, Mathematical Foundations of Imaging, Tomography and Wavefield Inversion, Cambridge University Press, 2012.

E. J. Candes, L. Demanet, The curvelet representation of wave propagators is optimally sparse, Communications on Pure and Applied Mathematics 58 (2005) 1472-1528.

R. J. Duffin, A. C. Schaeffer, A class of nonharmonic fourier series, Transactions of the American Mathematical Society 72 (1952) 341-366.

R. Feynman, R. Leighton, M. Sands, E. Hafner, The Feynman Lectures on Physics; Vol. I, volume 33, AAPT, 1965.

R. Courant, D. Hilbert, Methods of Mathematical Physics, volume 1, Wiley, New York, 1989. 
N. Ricker, The form and laws of propagation of seismic wavelets, Geophysics 18 (1953) 10-40.

E. Candes, L. Demanet, D. Donoho, L. Ying, Fast discrete curvelet transforms, Multiscale Modeling \& Simulation 5 (2006) 861-899. 